\title{
Integrating Krylov Deferred Correction and Generalized Finite Difference Methods for Dynamic Simulations of Wave Propagation Phenomena in Long-Time Intervals
}

\author{
Wenzhen $\mathrm{Qu}^{1,2, *}$, Hongwei $\mathrm{Gao}^{1}$ and Yan $\mathrm{Gu}^{1,2}$ \\ ${ }^{1}$ School of Mathematics and Statistics, Qingdao University, Qingdao, Shandong 266071, \\ China \\ ${ }^{2}$ Institute of Mechanics for Multifunctional Materials and Structures, Qingdao \\ University, Qingdao, Shandong 266071, China
}

Received 11 June 2020; Accepted (in revised version) 9 October 2020

\begin{abstract}
In this paper, a high-accuracy numerical scheme is developed for long-time dynamic simulations of 2D and 3D wave propagation phenomena. In the derivation of the present approach, the second order time derivative of the physical quantity in the wave equation is treated as a substitution variable. Based on the temporal discretization with the Krylov deferred correction (KDC) technique, the original wave problem is then converted into the modified Helmholtz equation. The transformed boundary value problem (BVP) in space is efficiently simulated by using the meshless generalized finite difference method (GFDM) with Taylor series after truncating the second and fourth order approximations. The developed scheme is finally verified by four numerical experiments including cases with complicated domains or the temporally piecewise defined source function. Numerical results match with the analytical solutions and results by the COMSOL software, which demonstrates that the developed KDC-GFDM can allow large time-step sizes for wave propagation problems in longtime intervals.
\end{abstract}

AMS subject classifications: 35L05, 65M06

Key words: Wave equation, Krylov deferred correction technique, large time-step, long-time simulation, generalized finite difference method.

\section{Introduction}

The wave equation is a very momentous partial differential equation (PDE) involved in

*Corresponding author.

Email: quwz@qdu.edu.cn (W. Qu) 
many physical applications, such as vibrations in strings and propagations of acoustic waves and water waves. The development of accurate and efficient numerical methods for simulations of wave propagation phenomena is an active research field [1-5]. Compared with mesh-dependent numerical algorithms, meshless methods, such as the method of fundamental solutions (MFS) [6-8], the method of approximate particular solutions (MAPS) [9,10], the boundary element-free method (BEFM) [11,12], and the singular boundary method (SBM) [13-19], have been one kind of powerful techniques because of no requirement of laborious mesh generation especially for 3D problems with complicated geometries.

Various meshless methods have so far been applied to the simulation of wave equation [20-23]. Dehghan and Shokri [20] introduced the radial basis functions (RBF) method to solve the one-dimensional (1D) wave equation with coupling $\theta$-weighted scheme. Zhang et al. [21] proposed an improved element-free Galerkin method (EFGM) for the damped wave propagation and discretized the time by the second-order center finite difference scheme. Based on the same technique of time discretization, Wang et al. [22] developed a central compact finite difference scheme for acoustic wave problems. Uren̈a et al. [23] applied the generalized finite difference method (GFDM) to the two-dimensional (2D) seismic wave propagation problem. The GFDM, as a very powerful meshless method, has been widely used for many applications [24-31] because of its simplicity, stability and easy implementation.

For time dependent wave problems, the finite difference scheme is a common choice for the discretization in temporal direction, such as those in [20-23]. However, the time step size has to be limited due to stability issues even for the five-stage Runge-Kutta method [32]. In long-time dynamic simulations, larger time step size is one of key factors to reduce the accumulation of the temporal-error. Several approaches were developed to allow larger time step size for temporal discretization, such as the spectral deferred correction (SDC) method [33,34], the integral deferred correction (InDC) method [35], and the Krylov deferred correction (KDC) method [36-39]. The KDC method applies the SDC as a preconditioner for the collocation formulations and then uses the NewtonKrylov technique to the solution of resulting equations, which reduces the number of iterations to converge.

By integrating advantages of the KDC technique and the meshless GFDM, a highaccuracy numerical approach called as the KDC-GFDM is constructed in this paper for dynamic simulations of $2 \mathrm{D}$ and $3 \mathrm{D}$ long-time wave propagation phenomena. For the developed algorithm, we consider the second order time derivative of the physical quantity in the wave equation as a substitution variable. Based on the temporal discretization with the KDC approach, the wave equation is then changed into the modified Helmholtz equation. The solution of the transformed boundary value problem (BVP) is determined by using the GFDM. The outline of this paper is as follows. The details of the developed algorithm are given in Section 2. 2D and 3D numerical examples including cases with the temporally piecewise defined source function or complicated domains are provided in Section 3. Some discussions are concluded in Section 4. 


\section{The numerical framework for wave equation}

We start from the following wave equation:

$$
\begin{array}{lll}
\frac{\partial^{2} u(\mathbf{x}, t)}{\partial t^{2}}=a^{2} \nabla^{2} u(\mathbf{x}, t)+f(\mathbf{x}, t), & \mathbf{x} \in \Omega, & t \geq 0, \\
u(\mathbf{x}, t)=g(\mathbf{x}, t), & \mathbf{x} \in \Gamma, \quad t \geq 0, \\
u(\mathbf{x}, 0)=u_{0}(\mathbf{x}), & \mathbf{x} \in \Omega, \quad t=0, \\
\frac{\partial u}{\partial t}(\mathbf{x}, 0)=v_{0}(\mathbf{x}), & \mathbf{x} \in \Omega, \quad t=0,
\end{array}
$$

where $u$ is the physical variable, $\nabla^{2}$ the spatial Laplacian operator, $f$ the source term, $\Omega$ the bounded domain in $R^{2}$ or $R^{3}$ with the boundary $\Gamma, \mathrm{x}$ the vector coordinate, $a$ the constant, $g, u_{0}, v_{0}$ the known functions.

\subsection{Krylov Deferred Correction (KDC) method}

The temporal pseudo-spectral discretization of the wave equation (2.1a) is first discussed in this subsection. A new unknown variable

$$
U(\mathbf{x}, t)=\frac{\partial^{2} u(\mathbf{x}, t)}{\partial t^{2}}
$$

is introduced to apply the KDC method. Based on this, Eq. (2.1a) can be then rewritten as

$$
U(\mathbf{x}, t)=a^{2} \nabla^{2}\left(u_{0}(\mathbf{x})+v_{0}(\mathbf{x}) t+\int_{0}^{t} \int_{0}^{\tau} U(\mathbf{x}, \zeta) d \zeta d \tau\right)+f(\mathbf{x}, t), \quad \mathbf{x} \in \Omega, \quad t \geq 0 .
$$

The size of time step is set to $\Delta t=\frac{T_{f}}{n}$ and $T_{f}, n$ are respectively the final time and number of time step. The details of temporal discretization of Eq. (2.2) is provided in the first time step, and the same process can be employed in the subsequent time steps. $p$ Gaussian nodes $\left\{t_{m}, m=1, \cdots, p\right\}$ are considered in $[0, \Delta t]$. Obviously, those nodes are linearly scaled. Time integrals of $U(\mathbf{x}, t)$ can be approximated by the following rectangular rule with the right end point

$$
\left\{\begin{array}{l}
\int_{t_{0}}^{t_{m}} U(\mathbf{x}, \zeta) d \zeta \approx \sum_{i=1}^{m} \Delta t_{i} U\left(\mathbf{x}, t_{i}\right) \\
\int_{t_{0}}^{t_{m}} \int_{0}^{\tau} U(\mathbf{x}, \zeta) d \zeta d \tau \approx \sum_{i=1}^{m} \Delta t_{i} \sum_{j=1}^{i} \Delta t_{j} U\left(\mathbf{x}, t_{j}\right)
\end{array}\right.
$$


where $m=1, \cdots, p$ and $\Delta t_{i}=t_{i}-t_{i-1},\left(t_{0}=0\right)$. Substituting Eq. (2.3) into Eq. (2.2), the following modified Helmholtz equation can be derived at each Gaussian node $t_{m}$

$$
\begin{aligned}
& \nabla^{2} U\left(\mathbf{x}, t_{m}\right)-\frac{1}{\left(a \Delta t_{m}\right)^{2}} U\left(\mathbf{x}, t_{m}\right) \\
= & \frac{-1}{\left(a \Delta t_{m}\right)^{2}}\left\{a ^ { 2 } \nabla ^ { 2 } \left(u_{0}(\mathbf{x})+v_{0}(\mathbf{x})\left(t_{m}-t_{0}\right)+\sum_{i=1}^{m-1} \Delta t_{i} \sum_{j=1}^{i} \Delta t_{j} U\left(\mathbf{x}, t_{j}\right)\right.\right. \\
& \left.\left.\quad+\Delta t_{m} \sum_{j=1}^{m-1} \Delta t_{j} U\left(\mathbf{x}, t_{j}\right)\right)+f\left(\mathbf{x}, t_{m}\right)\right\}
\end{aligned}
$$

with the boundary condition

$$
U\left(\mathbf{x}, t_{m}\right)=\frac{\partial^{2} g}{\partial t^{2}}\left(\mathbf{x}, t_{m}\right), \quad \mathbf{x} \in \Gamma .
$$

It will be easier to get the above general formulation by gradually making $t=t_{m},(m=$ $1, \cdots, p)$ in Eq. (2.2). An approximation of solution $\bar{U}\left(\mathbf{x}, t_{m}\right)$ of Eq. (2.4) is calculated by using the meshless GFDM, and the details will be provided in next section. After then, the results of $u$ are obtained as

$$
\left\{\begin{array}{l}
\overline{\mathbf{u}}_{t}(\mathbf{x})=\mathbf{v}_{0}(\mathbf{x})+\Delta t S \overline{\mathbf{U}}(\mathbf{x}), \\
\overline{\mathbf{u}}(\mathbf{x})=\mathbf{u}_{0}(\mathbf{x})+\Delta t S \overline{\mathbf{u}}_{t}(\mathbf{x}),
\end{array}\right.
$$

where

$$
\begin{array}{ll}
\overline{\mathbf{u}}(\mathbf{x})=\left[\bar{u}\left(\mathbf{x}, t_{1}\right), \bar{u}\left(\mathbf{x}, t_{2}\right), \cdots, \bar{u}\left(\mathbf{x}, t_{p}\right)\right]^{T}, & \overline{\mathbf{u}}_{t}(\mathbf{x})=\left[\bar{u}_{t}\left(\mathbf{x}, t_{1}\right), \bar{u}_{t}\left(\mathbf{x}, t_{2}\right), \cdots, \bar{u}_{t}\left(\mathbf{x}, t_{p}\right)\right]^{T}, \\
\mathbf{u}_{0}(\mathbf{x})=\left[u_{0}(\mathbf{x}), u_{0}(\mathbf{x}), \cdots, u_{0}(\mathbf{x})\right]^{T}, & \mathbf{v}_{0}(\mathbf{x})=\left[v_{0}(\mathbf{x}), v_{0}(\mathbf{x}), \cdots, v_{0}(\mathbf{x})\right]^{T}, \\
\overline{\mathbf{U}}(\mathbf{x})=\left[\bar{U}\left(\mathbf{x}, t_{1}\right), \bar{U}\left(\mathbf{x}, t_{2}\right), \cdots, \bar{U}\left(\mathbf{x}, t_{p}\right)\right]^{T}, &
\end{array}
$$

$S$ denotes the spectral integration matrix [37] and $\bar{u}_{t}$ means the first order time derivative of $\bar{u}$. It is worth mentioning that $S$ can be precomputed owing to its independence of the time step size $\Delta t$.

To improve the accuracy of numerical results, the equation related with the error $\delta(\mathbf{x}, t)=U(\mathbf{x}, t)-\bar{U}(\mathbf{x}, t)$ is defined as follows

$$
\begin{aligned}
\bar{U}(\mathbf{x}, t)+\delta(\mathbf{x}, t)=a^{2} \nabla^{2} & \left(u_{0}(\mathbf{x})+v_{0}(\mathbf{x}) t+\int_{0}^{t} \int_{0}^{\tau} \bar{U}(\mathbf{x}, \zeta) d \zeta d \tau\right. \\
& \left.+\int_{0}^{t} \int_{0}^{\tau} \delta(\mathbf{x}, \zeta) d \zeta d \tau\right)+f(\mathbf{x}, t)
\end{aligned}
$$

where $\bar{U}(\mathbf{x}, t)$ is constructed based on the polynomial interpolation of $\overline{\mathbf{U}}(\mathbf{x})$. We use the rectangular rule for $\int_{0}^{t} \int_{0}^{\tau} \delta(\mathbf{x}, \zeta) d \zeta d \tau$ and formulation (2.5) to transform Eq. (2.6) as the 
following equation at each Gaussian node $t_{m}$

$$
\begin{aligned}
& \nabla^{2} \delta\left(\mathbf{x}, t_{m}\right)-\frac{1}{\left(a \Delta t_{m}\right)^{2}} \delta\left(\mathbf{x}, t_{m}\right) \\
= & \frac{1}{\left(a \Delta t_{m}\right)^{2}}\left\{\bar{U}\left(\mathbf{x}, t_{m}\right)-a^{2} \nabla^{2}\left(\bar{u}\left(\mathbf{x}, t_{m}\right)+\sum_{i=1}^{m-1} \Delta t_{i} \sum_{j=1}^{i} \Delta t_{j} \delta\left(\mathbf{x}, t_{j}\right)\right.\right. \\
& \left.\left.\quad+\Delta t_{m} \sum_{j=1}^{m-1} \Delta t_{j} \delta\left(\mathbf{x}, t_{j}\right)\right)-f\left(\mathbf{x}, t_{m}\right)\right\},
\end{aligned}
$$

with $\delta\left(\mathbf{x}, t_{m}\right)=0$ as the boundary condition. Obviously, Eq. (2.7) is also a modified Helmholtz equation as same as Eq. (2.4). After then, we can define a vector

$$
\bar{\delta}(\mathbf{x})=\left[\bar{\delta}\left(\mathbf{x}, t_{1}\right), \bar{\delta}\left(\mathbf{x}, t_{2}\right), \cdots, \bar{\delta}\left(\mathbf{x}, t_{p}\right)\right]^{T}
$$

based on the results $\bar{\delta}(\mathbf{x}, t)$ of Eq. (2.7) calculated by the meshless GFDM.

In the SDC method, $\bar{\delta}+\overline{\mathbf{U}}$ is directly treated as an updated solution, and the iterative process does not stop until a prescribed error tolerance is achieved. However, in the KDC method, Eq. (2.7) is considered as a function $H(\overline{\mathbf{U}})=\bar{\delta}$. High-accuracy results $\overline{\mathbf{U}}$ for satisfying Eq. (2.2) can be obtained when $\bar{\delta}$ is equal to zero vector. That is to say that the solution of the collocation formulation in Eq. (2.5) is equivalent to solving $H(\overline{\mathbf{U}})=$ 0 . We solve $H(\overline{\mathbf{U}})=0$ by the Jacobian-free Newton-Krylov (JFNK) solvers [40]. Finally, assuming solution is fully resolved in spatial direction, the resulting scheme has order $2 p$ when $p$ Gaussian nodes are used.

\subsection{Generalized Finite Difference Method (GFDM)}

In the derivation of the KDC technique, both $U\left(\mathbf{x}, t_{m}\right)$ in Eq. (2.4) and

$$
\delta\left(\mathbf{x}, t_{m}\right)
$$

in Eq. (2.7) satisfy the following equation

$$
\nabla^{2} \phi(\mathbf{x})-\frac{1}{\left(a \Delta t_{m}\right)^{2}} \phi(\mathbf{x})=Q(\mathbf{x}), \quad \mathbf{x} \in \Omega,
$$

with the boundary condition

$$
\phi(\mathbf{x})=\bar{\phi}(\mathbf{x}), \quad \mathbf{x} \in \Gamma,
$$

in which $\phi(\mathbf{x})=U\left(\mathbf{x}, t_{m}\right)$ or $\phi(\mathbf{x})=\delta\left(\mathbf{x}, t_{m}\right), a$ is the constant in Eq. (2.1a), $\Delta t_{m}=t_{m}-t_{m-1}$, $Q(\mathbf{x})$ is known as shown in the right hand side of Eq. (2.4) or Eq. (2.7). For the boundary condition, we should note that

$$
\bar{\phi}(\mathbf{x})=\frac{\partial^{2} g}{\partial t^{2}}\left(\mathbf{x}, t_{m}\right) \quad \text { for } U\left(\mathbf{x}, t_{m}\right)
$$




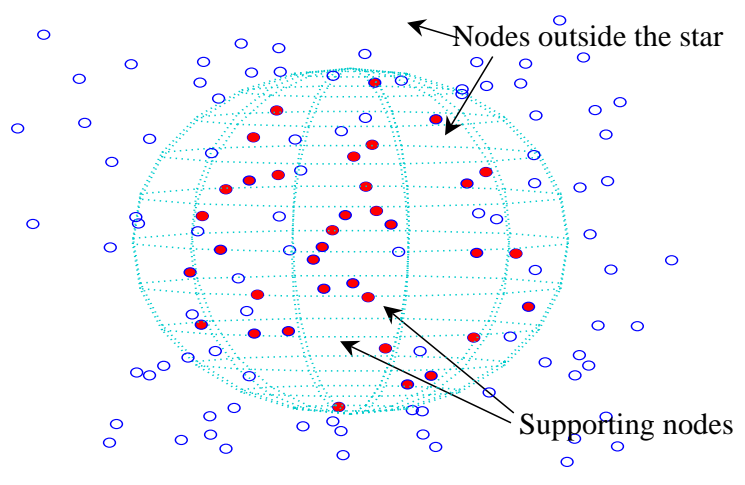

Figure 1: Collocation points and stars.

or

$$
\bar{\phi}(\mathbf{x})=0 \quad \text { for } \delta\left(\mathbf{x}, t_{m}\right)
$$

on the boundary.

Next, we show the details of the solution of Eqs. (2.8) and (2.9) in 3D case by using the GFDM. The implementations of the GFDM for solving the above-mentioned 2D system are similar, and thus these are neglected in this paper. For the GFDM, collocation points (see Fig. 1) are first distributed in $\Omega$ with its boundary. For each node $x_{0}$ (called as the center node), we then define a star containing $m$ nearest nodes $\mathbf{x}_{i},(i=1, \cdots, m)$ (i.e., $\mid x_{i}-$ $\left.x_{0} \mid \leq d_{m}\right)$, which are treated as the supporting nodes.

Assume $\phi_{i}=\phi\left(\mathbf{x}_{i}\right),(i=0,1, \cdots, m)$, and all nodes $\left(\mathbf{x}_{i}\right)_{i=1}^{m}$ are inside the star of the central node $\mathbf{x}_{0}$. For this star, we have the following Taylor series expansions of $\phi_{i}$ around $\mathbf{x}_{0}$ [41]

$$
\begin{aligned}
\phi_{i}=\phi_{0} & +h_{i} \frac{\partial \phi_{0}}{\partial x_{1}}+k_{i} \frac{\partial \phi_{0}}{\partial x_{2}}+l_{i} \frac{\partial \phi_{0}}{\partial x_{3}}+\frac{1}{2}\left(h_{i}^{2} \frac{\partial^{2} \phi_{0}}{\partial x_{1}^{2}}+k_{i}^{2} \frac{\partial^{2} \phi_{0}}{\partial x_{2}^{2}}+l_{i}^{2} \frac{\partial^{2} \phi_{0}}{\partial x_{3}^{2}}\right) \\
& +h_{i} k_{i} \frac{\partial^{2} \phi_{0}}{\partial x_{1} \partial x_{2}}+h_{i} l_{i} \frac{\partial^{2} \phi_{0}}{\partial x_{1} \partial x_{3}}+k_{i} l_{i} \frac{\partial^{2} \phi_{0}}{\partial x_{2} \partial x_{3}}+\cdots, \quad i=1, \cdots, m,
\end{aligned}
$$

with

$$
h_{i}=x_{i}^{1}-x_{0}^{1}, \quad k_{i}=x_{i}^{2}-x_{0}^{2}, \quad l_{i}=x_{i}^{3}-x_{0}^{3},
$$

where $x_{0}^{j},(j=1,2,3)$ and $x_{i}^{j},(j=1,2,3)$ are the coordinates of $x_{0}$ and $x_{i}$ respectively.

We truncate the Taylor series in Eq. (2.10) after the second-order derivatives to construct a residual function $B(\phi)$ as follows

$$
\begin{aligned}
B(\phi)=\sum_{i=1}^{m} & {\left[\left(\phi_{0}-\phi_{i}+h_{i} \frac{\partial \phi_{0}}{\partial x_{1}}+k_{i} \frac{\partial \phi_{0}}{\partial x_{2}}+l_{i} \frac{\partial \phi_{0}}{\partial x_{3}}+\frac{h_{i}^{2}}{2} \frac{\partial^{2} \phi_{0}}{\partial x_{1}^{2}}+\frac{k_{i}^{2}}{2} \frac{\partial^{2} \phi_{0}}{\partial x_{2}^{2}}+\frac{l_{i}^{2}}{2} \frac{\partial^{2} \phi_{0}}{\partial x_{3}^{2}}\right.\right.} \\
+ & \left.\left.h_{i} k_{i} \frac{\partial^{2} \phi_{0}}{\partial x_{1} \partial x_{2}}+h_{i} l_{i} \frac{\partial^{2} \phi_{0}}{\partial x_{1} \partial x_{3}}+k_{i} l_{i} \frac{\partial^{2} \phi_{0}}{\partial x_{2} \partial x_{3}}\right) \omega_{i}\right]^{2}
\end{aligned}
$$


with the weighting function $\omega_{i}[42]$ given as

$$
\omega_{i}=\frac{\exp \left[-\left(d_{i}\right)^{2}\right]-\exp \left[-\left(d_{m}\right)^{2}\right]}{1-\exp \left[-\left(d_{m}\right)^{2}\right]}, \quad i=1, \cdots, m,
$$

where $d_{i}=\left|x_{i}-x_{0}\right|$ represents the distance between $x_{0}$ and $x_{i}, d_{m}=\max \left\{d_{i}, i=1, \cdots, m\right\}$. We also refer interested readers to [43-46] for some other choices.

To construct a second order partial differential equation (PDE) at the central node $x_{0}$, we assume a vector of partial derivatives as

$$
D_{\phi}=\left[\frac{\partial \phi_{0}}{\partial x_{1}}, \frac{\partial \phi_{0}}{\partial x_{2}}, \frac{\partial \phi_{0}}{\partial x_{3}}, \frac{\partial^{2} \phi_{0}}{\partial x_{1}^{2}}, \frac{\partial^{2} \phi_{0}}{\partial x_{2}^{2}}, \frac{\partial^{2} \phi_{0}}{\partial x_{3}^{2}}, \frac{\partial^{2} \phi_{0}}{\partial x_{1} \partial x_{2}}, \frac{\partial^{2} \phi_{0}}{\partial x_{1} \partial x_{3}}, \frac{\partial^{2} \phi_{0}}{\partial x_{2} \partial x_{3}}\right]^{T} .
$$

Minimizing function $B(\phi)$ with respect to $D_{\phi}$, i.e.,

$$
\frac{\partial B(\phi)}{\partial\left\{D_{\phi}\right\}}=0
$$

we can have a system of linear equations

$$
A D_{\phi}=b
$$

where

$$
A=\sum_{i=1}^{m} \operatorname{diag}\left(E_{1}^{(i)}\right) \coprod \operatorname{diag}\left(E_{2}^{(i)}\right)
$$

represents a symmetric matrix, $\amalg$ is a $9 \times 9$ matrix with each element equals to one, $\operatorname{diag}\left(E_{1}^{(i)}\right)$ and $\operatorname{diag}\left(E_{2}^{(i)}\right)$ respectively denote two diagonal matrices with diagonal elements as

$$
\begin{aligned}
& E_{1}^{(i)}=\omega_{i}\left[\begin{array}{lllllllll}
h_{i} & k_{i} & l_{i} & h_{i}^{2} & k_{i}^{2} & l_{i}^{2} & h_{i} k_{i} & h_{i} l_{i} & k_{i} l_{i}
\end{array}\right]^{T}, \\
& E_{2}^{(i)}=\frac{\omega_{i}}{2}\left[\begin{array}{lllllllll}
2 h_{i} & 2 k_{i} & 2 l_{i} & h_{i}^{2} & k_{i}^{2} & l_{i}^{2} & 2 h_{i} k_{i} & 2 h_{i} l_{i} & 2 k_{i} l_{i}
\end{array}\right]^{T},
\end{aligned}
$$

and $b$ is given as

$$
b=B \Phi=\left(-\sum_{i=1}^{m} \omega_{i} E_{2}^{(i)}, \omega_{1} E_{2}^{(1)}, \omega_{2} E_{2}^{(2)}, \cdots, \omega_{m} E_{2}^{(m)}\right)_{9 \times(m+1)}\left(\begin{array}{c}
\phi_{0} \\
\phi_{1} \\
\phi_{2} \\
\vdots \\
\phi_{m}
\end{array}\right)_{(m+1) \times 1},
$$


in which $\Phi=\left[\phi_{0}, \phi_{1}, \phi_{2}, \cdots, \phi_{m}\right]^{T}$ are values of $\phi(\mathbf{x})$ at nodes inside the star. By using Eqs. (2.16)-(2.19), we can express $D_{\phi}$ as

$$
D_{\phi}=A^{-1} b=A^{-1} B \Phi=E\left(\begin{array}{c}
\phi_{0} \\
\phi_{1} \\
\phi_{2} \\
\vdots \\
\phi_{m}
\end{array}\right)
$$

where $E=A^{-1} B$. As we can see from Eq. (2.20), the derivatives $D_{\phi}$ of $\phi$ at $x_{0}$ have been expressed as linear combinations of function values $\Phi=\left[\phi_{0}, \phi_{1}, \phi_{2}, \cdots, \phi_{m}\right]^{T}$. Then, based on Eq. (2.20), the left-hand of the modified Helmholtz equation (2.8) at node $\mathbf{x}_{0}$ is rewritten as

$$
\nabla^{2} \phi_{0}-\frac{1}{\left(a \Delta t_{i}\right)^{2}} \phi_{0}=m_{0} \phi_{0}+\sum_{i=1}^{m} m_{i} \phi_{i}
$$

where $m_{i},(i=0,1, \cdots, m)$ are determined based on Eq. (2.20).

We can obtain a similar equation at each collocation point in $\Omega$, and then form a system of linear algebraic equations by employing the boundary conditions simultaneously. At any interior collocation point, the corresponding row of the matrix of this linear system only has no more than $m+1$ non-zero elements based on Eq. (2.20). That is to say that the equation system of the GFDM has a sparse matrix and thus is suitable for the solution of large-scale problems. We use the iterative solver of the generalized minimal residual method (GMRES) to solve this equation system, which has $\mathcal{O}(N)$ computational complexities and $N$ is the dimension of the sparse matrix.

It can be easily demonstrated that the GFDM has higher accuracy when truncating Eq. (2.10) after higher-order derivatives. In this work, we truncate Eq. (2.10) after the second-order and fourth-order derivatives to construct the function $B(\phi)$, and call the method as the GFDM(2) and the GFDM(4) respectively. The derivation of the GFDM(4) is very similar with that of the GFDM(2) which is therefore neglected. Some other meshless collocation methods, such as the finite point method [47], the element-free Galerkin method [48,49], and the localized collocation Trefftz method [50], can also be used in a similar way for the problem of Eqs. (2.8) and (2.9).

\section{Numerical experiments}

In this section, we provide four numerical examples including 2D and 3D wave problems to verify the accuracy, convergency, and stability of the developed approach. The 
formulation for numerical error estimation is given as [51]:

$$
\text { Global Error }(\mathrm{GE})=\sqrt{\sum_{k=1}^{\bar{N}}\left(u_{\text {numerical }}^{k}-u_{\text {exact }}^{k}\right)^{2}} / \sqrt{\sum_{k=1}^{\bar{N}}\left(u_{\text {exact }}^{k}\right)^{2}} \text {, }
$$

where $u_{\text {numerical }}^{k}$ and $u_{\text {exact }}^{k}$ respectively represent the numerical and analytical results, and $\bar{N}$ denotes the number of test points. The convergence rate (CA) of the developed method is formulated as

$$
\mathrm{CA}=\left|\frac{\ln \left(\mathrm{GE}_{2} / \mathrm{GE}_{1}\right)}{\ln \left(\xi_{1} / \xi_{2}\right)}\right|
$$

where $\xi_{i},(i=1,2)$ denote the numbers of collocation points or the time step sizes, and $\mathrm{GE}_{i},(i=1,2)$ represent the global errors with respect to $\xi_{i},(i=1,2)$.

We assume the number of supporting nodes to be $m=12$ for the GFDM(2) and $m=27$ for the GFDM(4) in 2D cases, and $m=20$ for the GFDM(2) and $m=60$ for the GFDM(4) in $3 \mathrm{D}$ cases. In addition, the coordinates of collocation points were obtained by using the HyperMesh software.

\subsection{D wave propagation in a unit square}

We consider the wave propagation in a unit square with side length $0 \leq x, y \leq 1$ under the system of equations as follows:

$$
\begin{array}{lll}
\frac{\partial^{2} u(x, y, t)}{\partial t^{2}}=\frac{1}{2} \nabla^{2} u(x, y, t), & (x, y) \in \Omega, & t \geq 0, \\
u(x, y, t)=0, & (x, y) \in \Gamma, & t \geq 0, \\
u(x, y, 0)=\sin (\pi x) \sin (\pi y), & (x, y) \in \Omega, & t=0, \\
\frac{\partial u}{\partial t}(x, y, 0)=0, & (x, y) \in \Omega, & t=0 .
\end{array}
$$

The exact solution for this case is given by

$$
u(x, y, t)=\sin (\pi x) \sin (\pi y) \cos (\pi t) .
$$

The solution is first simulated from $t=0$ to $t=5$, and time step size is set to $\Delta t=1$. Fig. 2 shows variation curves of global errors of numerical results at all collocation points by using the KDC-GFDM(2) and the KDC-GFDM(4) with different Gaussian node number $p$ in $\Delta t$ and collocation point number $n$ in square domain. We can observe from the figure that the present approach obtains convergence solutions at $t=5$ when using more Gaussian nodes or collocation points. Moreover, it can be found that higher-accuracy results are obtained by the KDC-GFDM(4) compared with those by the KDC-GFDM(2). Based on Eq. (3.2), the convergence rate of the KDC-GFDM(4) in space is 2.06 as shown in Fig. 2(b). 


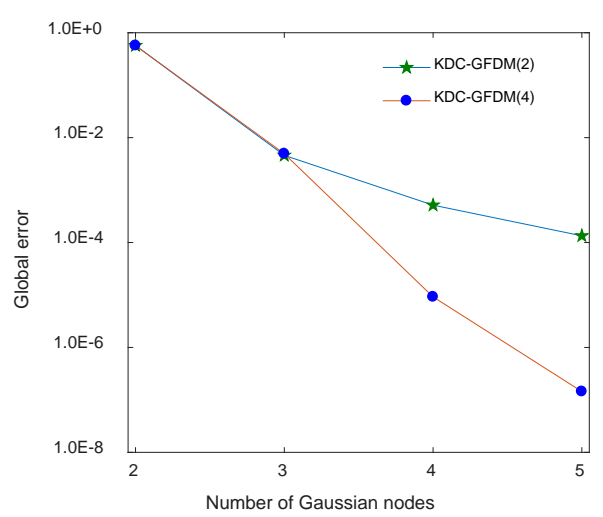

(a)

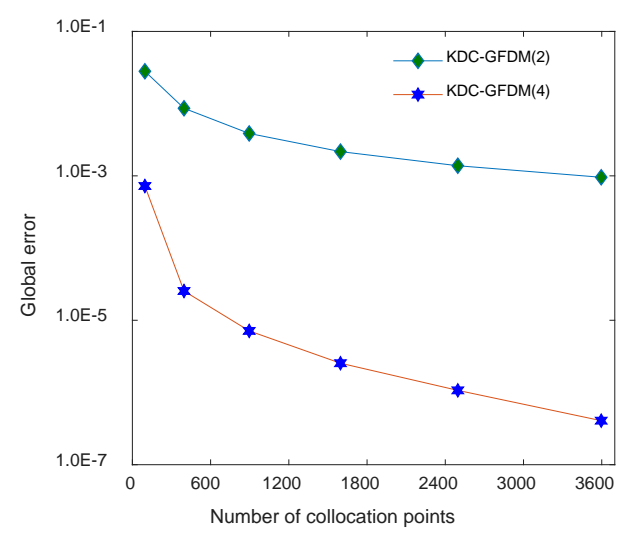

(b)

Figure 2: Global errors obtained by the developed approach with: (a) the different number $p(n=6396)$ or (b) the different number $n(p=5)$.

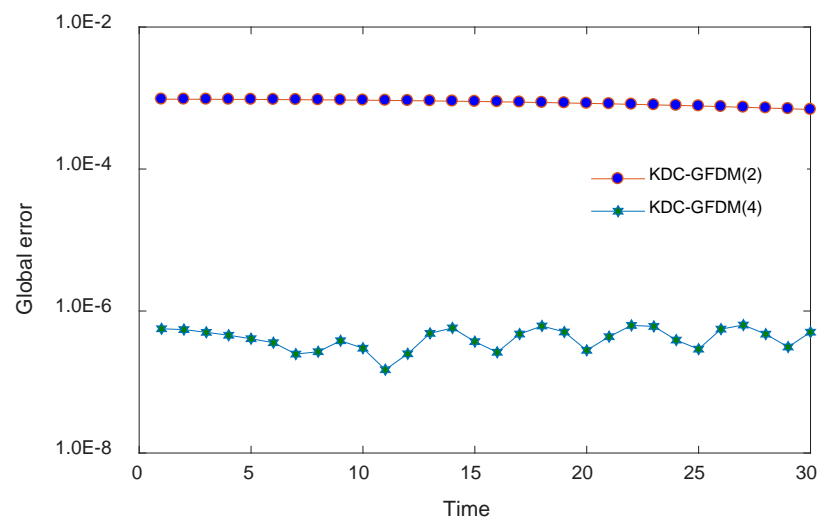

Figure 3: Global error variations of the developed method for the solution in time interval $t \in[0,30]$ (Dirichlet boundary condition).

We rerun the simulation in time interval $t \in[0,30]$ and assume the time step size to be $\Delta t=1$. In each time step $\Delta t, 5$ Gaussian nodes are employed. 3596 collocation points are distributed in space. Fig. 3 provides global errors of numerical results at all collocation points as a function of time. It can be observed that errors of the KDC-GFDM(2) and the KDC-GFDM(4) are less than 2E-03 and 1E-06. In addition, the global errors of these two approaches remain stable as the time marching process, which is a good feature for dynamic simulations in long time intervals. The reason of this phenomenon is that the energy is conserved when using the resulting collocation formulation of the present method with Gaussian nodes, which is called as a symplectic scheme [52].

Finally, we impose the Neumann boundary condition $-\pi \sin (\pi y)$ on the boundaries 


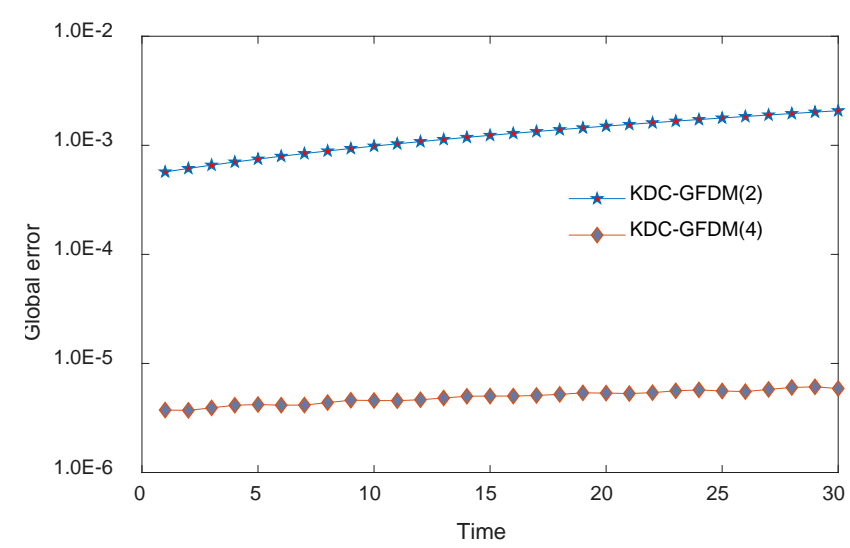

Figure 4: Global error variations of the developed method for the solution in time interval $t \in[0,30]$ (Mixed boundary condition).

$x=0$ and $x=1$ to replace the original Dirichlet boundary condition, and remain the same time step size, Gaussian nodes, and collocation points as before. Fig. 4 plots global errors of numerical results at all collocation points as a function of time. As we can see, global errors for the mixed boundary condition are slightly larger than those in Fig. 3 for the Dirichlet boundary condition.

\subsection{D wave propagation in a complicated domain}

We consider the wave propagation in a 2D complicated domain of Fig. 5(a) as the second numerical experiment

$$
\begin{array}{lll}
\frac{\partial^{2} u(x, y, t)}{\partial t^{2}}=115600 \nabla^{2} u(x, y, t) & \\
\quad+e^{x+y} e^{\cos t}\left(\sin ^{2} t-\cos t-231200\right), & (x, y) \in \Omega, & t \geq 0, \\
u(x, y, t)=e^{x+y} e^{\cos t}, & (x, y) \in \Gamma, & t \geq 0, \\
u(x, y, 0)=e^{x+y+1}, & (x, y) \in \Omega, & t=0, \\
\frac{\partial u}{\partial t}(x, y, 0)=0, & (x, y) \in \Omega, & t=0 .
\end{array}
$$

The exact solution is determined as $u(x, y, t)=e^{x+y} e^{\cos t}$. Fig. $5(\mathrm{~b})$ shows the distribution of 3398 collocation points and the dimension $2.7 \times 1.4$ of the domain.

We assume the final time to be $t=12$ and the size of time step to be $\Delta t=1.2$. Fig. 6 displays the contours of relative errors of numerical results at all interior collocation points by using the KDC-GFDM(2) with different numbers $n$ of collocation points and $p$ of Gaussian nodes. As it can be seen, the max relative error is less than 7E-03 even using the 


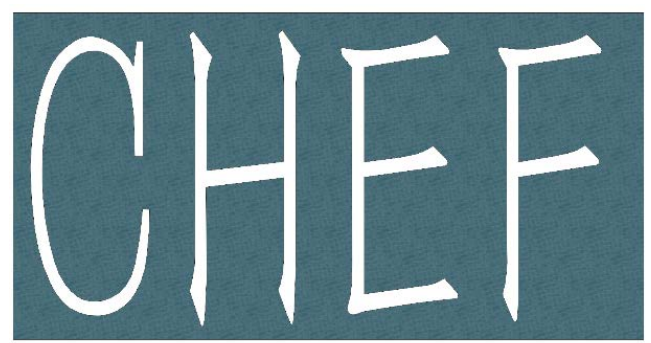

(a)

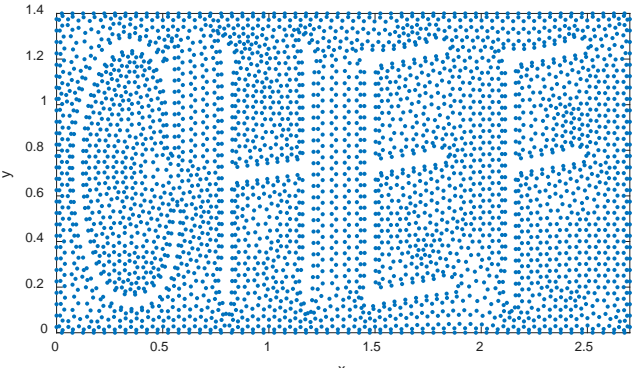

(b)

Figure 5: A 2D complicated domain (a) and 3398 collocation points in space (b).

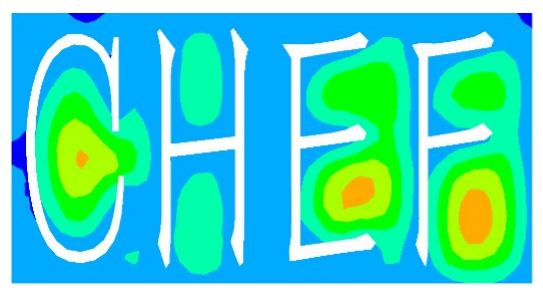

(a) $n=1916, p=3$

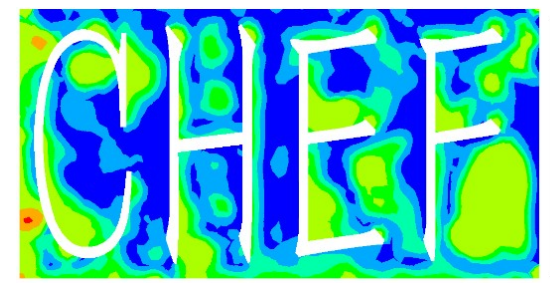

(c) $n=5266, p=5$
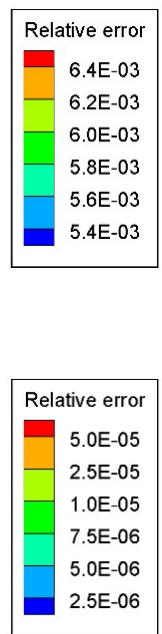

$5 \mathrm{E}-06$

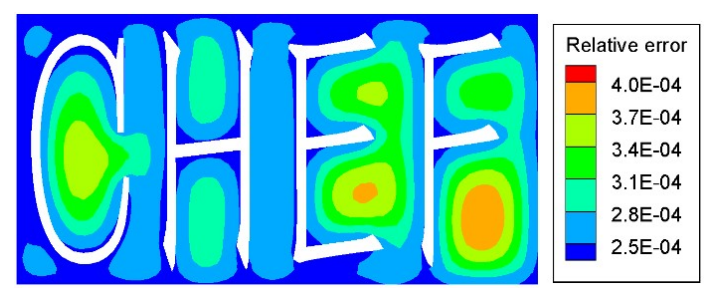

(b) $n=3398, p=4$

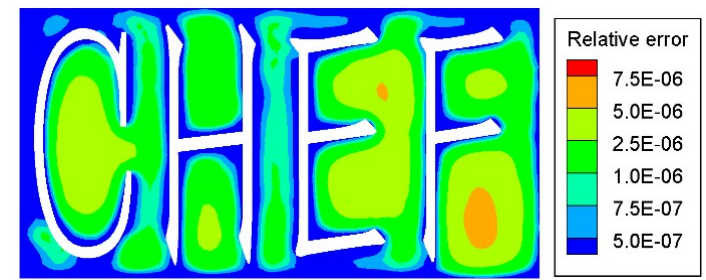

(d) $n=8918, p=6$

Figure 6: Relative error surfaces of results in the computational domain with different $n, p$.

present approach with $n=1916$ and $p=3$. We also can observe that the errors decay rapidly as a function of $n$ and $p$.

A simulation of wave propagation in a long-time interval $t \in[0,1000]$ is analyzed by the developed approach. The size of time step is assumed to be $\Delta t=1$, and 5266 collocation points are distributed in the computational domain. Fig. 7 plots global error variations of $u$ at 5266 collocation points by using the KDC-GFDM(2) with 6 Gaussian nodes and the KDC-GFDM(4) with 8 Gaussian nodes in each time step. We can see that the errors of the present method have a little up-and-down motion as a function of time but no increase in general. 


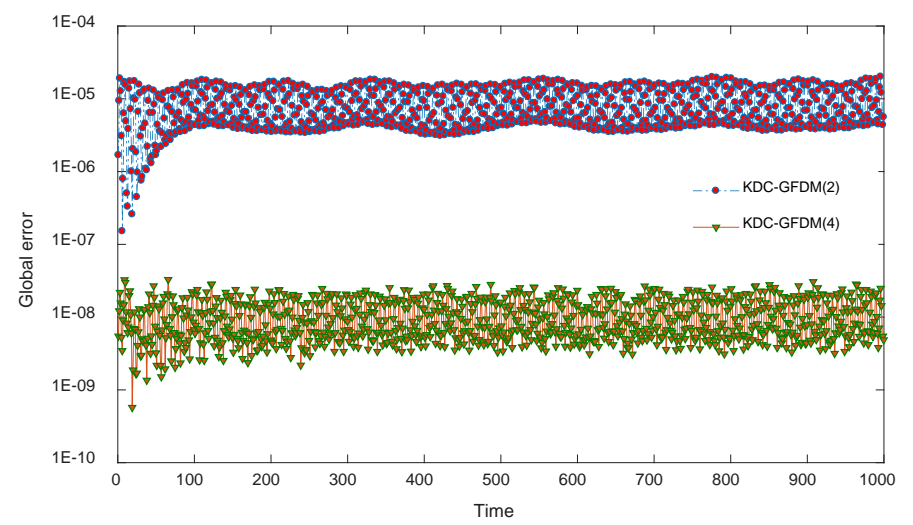

Figure 7: The global error variations of numerical results from $t=0$ to $t=1000$.

\subsection{D wave propagation in a 3D sawtooth structure (thin body)}

The 3D wave propagation in a 3D sawtooth structure as shown in Fig. 8(a) is considered. The dimension of the serrated structure is $1.0 \times 0.3 \times 0.033$. The system of wave equation is given by

$$
\begin{aligned}
& \frac{\partial^{2} u(x, y, z, t)}{\partial t^{2}}=100 \nabla^{2} u(x, y, z, t)+100\left(\sin x-e^{y}\right) \sin (\cos t) \\
& -\left(\sin x+e^{y}+z\right)\left[\cos (\cos t) \cos t+\sin (\cos t) \sin ^{2} t\right], \\
& (x, y, z) \in \Omega, \quad t \geq 0, \\
& u(x, y, z, t)=\left(\sin x+e^{y}+z\right) \sin (\cos t), \quad(x, y, z) \in \Gamma, \quad t \geq 0, \\
& u(x, y, z, 0)=\left(\sin x+e^{y}+z\right) \sin 1, \quad(x, y, z) \in \Omega, \quad t=0, \\
& \frac{\partial u}{\partial t}(x, y, z, 0)=0 \text {, } \\
& (x, y, z) \in \Omega, \quad t=0 \text {. }
\end{aligned}
$$

The exact solution is expressed as

$$
u(x, y, z, t)=\left(\sin x+e^{y}+z\right) \sin (\cos t) .
$$

In this case, the present method uses 1924 collocation points (see Fig. 8(b)) in space and 3 Gaussian nodes in the time step to simulate the solution in time interval $t \in[0,2]$. The curves of global errors of $u$ at interior collocation points are plotted in Fig. 9. As it is observed, the KDC-GFDM(4) shows a fast convergence as a function of time step size. Based on Eq. (3.2), the convergence rate of the KDC-GFDM(4) in time is 6.73. In addition, the KDC-GFDM(2) also has a good performance until the method is limited to the precision of the GFDM(2) in the space simulation. By setting the time step size $\Delta t=1$ and 5 Gaussian nodes in $\Delta t$, we rerun the code to verify the performance of the present method for long-time 3D wave propagation from $t=0$ to $t=200$. Fig. 10 plots the global 


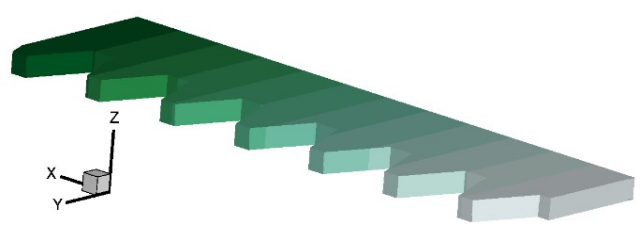

(a)

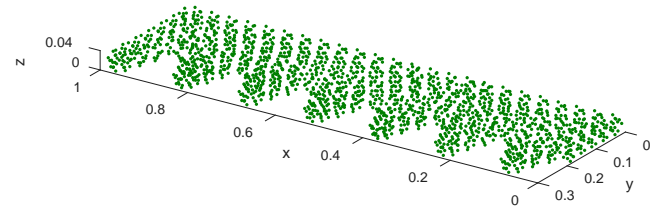

(b)

Figure 8: 3D sawtooth structure (a) and 1924 collocation points in space (b).

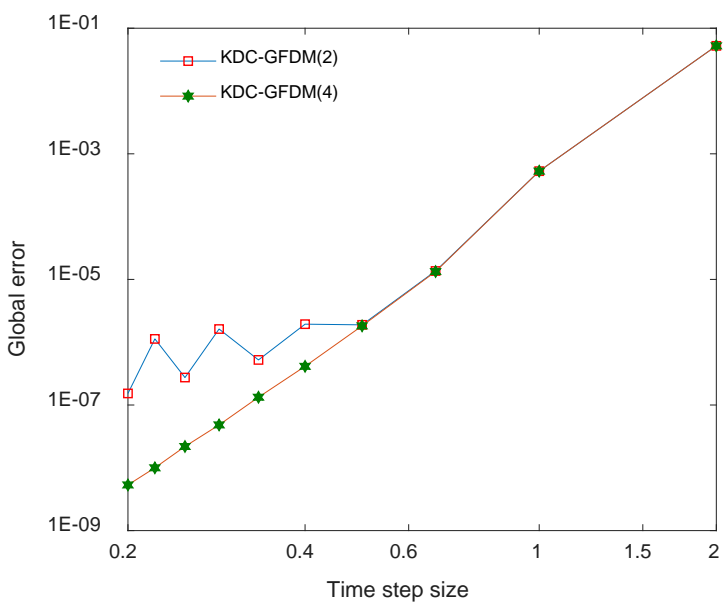

Figure 9: Curves of global errors of numerical results as a function of time step size.

error curves of numerical results at all interior collocation points from $t=0$ to $t=200$. It can be found that errors of the developed method are relatively stable as a function of time.

Next, the solution is simulated in time interval $t \in[0,20]$. The time step size is set to $\Delta t=2$ and 8 Gaussian nodes are used in $\Delta t$. Fig. 11 displays relative error contours of numerical results of fluxes on the surface of sawtooth structure calculated by the KDCGFDM(2). As we can observe from this figure, the developed method obtains the satisfied results at final time $t=20$. Table 1 lists the condition numbers $(\mathrm{CN})$ of the GFDM(2) for solving Eqs. (2.8) and (2.9) with respect to $\Delta t_{m},(m=1, \cdots, p)$.

Table 1: The condition numbers of the GFDM(2) for solving Eqs. (2.8) and (2.9).

\begin{tabular}{||c|cccccccc||}
\hline$\Delta t_{m}$ & $\Delta t_{1}$ & $\Delta t_{2}$ & $\Delta t_{3}$ & $\Delta t_{4}$ & $\Delta t_{5}$ & $\Delta t_{6}$ & $\Delta t_{7}$ & $\Delta t_{8}$ \\
\hline $\mathrm{CN}$ & $2.486 \mathrm{E}+05$ & $2.486 \mathrm{E}+05$ & $2.486 \mathrm{E}+05$ & $2.487 \mathrm{E}+05$ & $2.487 \mathrm{E}+05$ & $2.487 \mathrm{E}+05$ & $2.486 \mathrm{E}+05$ & $2.486 \mathrm{E}+05$ \\
\hline
\end{tabular}




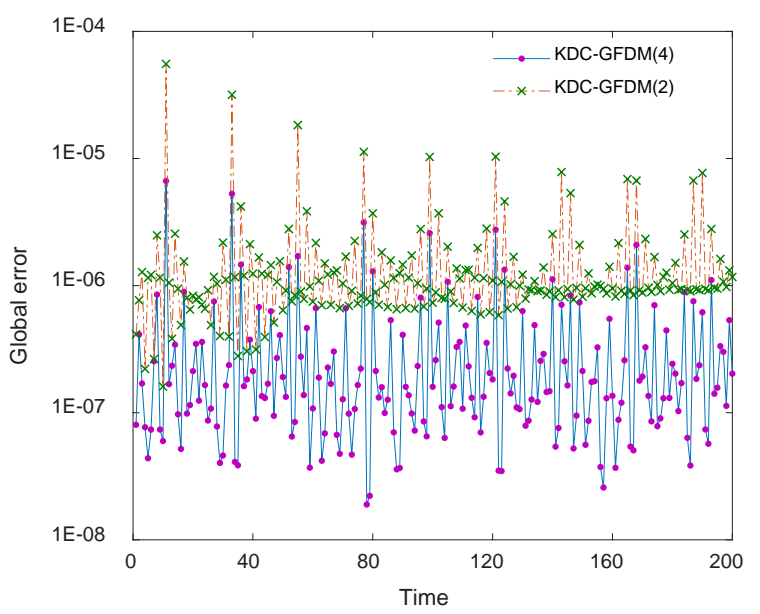

Figure 10: Curves of global errors of numerical results as a function of time.

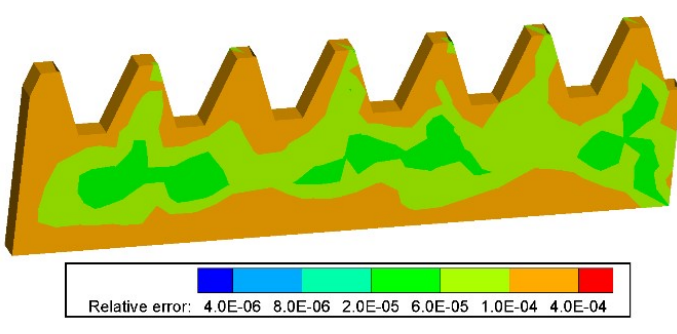

(a) $\partial u / \partial x$

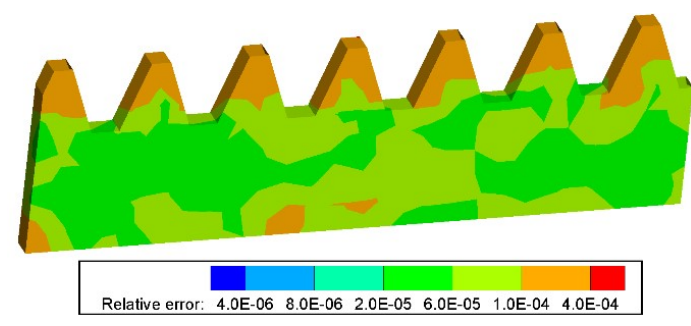

(c) $\partial u / \partial z$

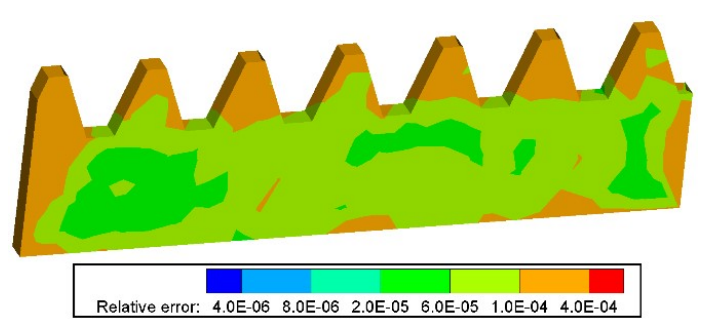

(b) $\partial u / \partial y$

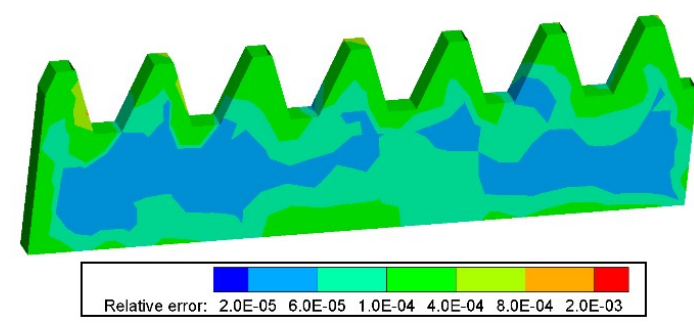

(d) $\partial u / \partial \mathbf{n}$

Figure 11: Relative error contours of numerical results on the boundary obtained by the KDC-GFDM(2).

\subsection{D wave propagation in a mechanical component}

The 3D wave propagation in a mechanical component (see Fig. 12(a)) with a complicated domain is considered. The dimension of this mechanical component is 1.68 in $x$ direction, 


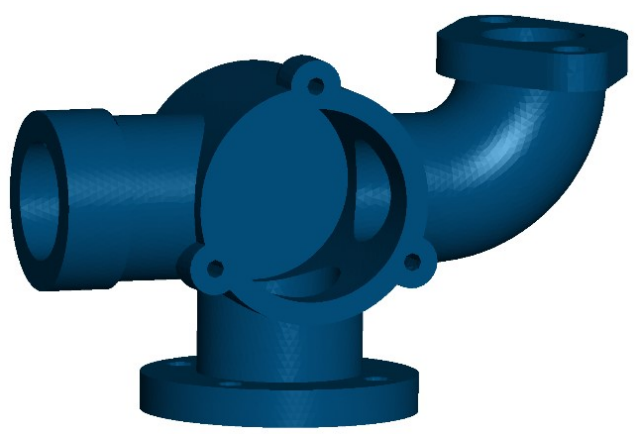

(a)

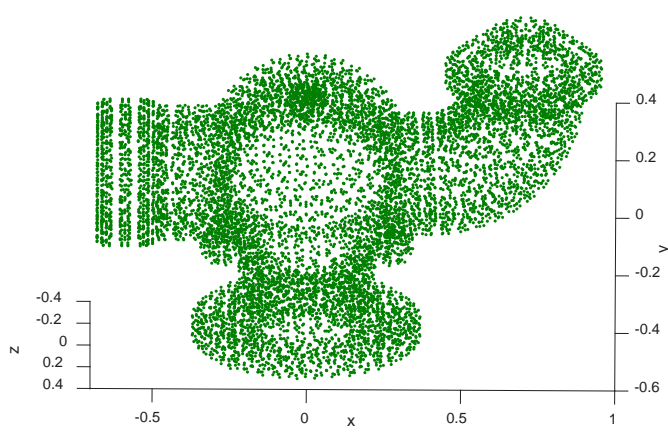

(b)

Figure 12: 3D mechanical component (a) and 11081 collocation points in space (b).

0.98 in $y$ direction, and 0.79 in $z$ direction. The system of wave equation is

$$
\begin{array}{lll}
\frac{\partial^{2} u(x, y, z, t)}{\partial t^{2}}=100 \nabla^{2} u(x, y, z, t)+f(x, y, z, t), & (x, y, z) \in \Omega, & t \geq 0 \\
u(x, y, z, t)=e^{x+y+z} \cos t, & (x, y, z) \in \Gamma, & t \geq 0 \\
u(x, y, z, 0)=e^{x+y+z}, & (x, y, z) \in \Omega, & t=0 \\
\frac{\partial u}{\partial t}(x, y, z, 0)=0, & (x, y, z) \in \Omega, & t=0 .
\end{array}
$$

where

$$
f(x, y, z, t)= \begin{cases}10 e^{-\frac{t}{10}}, & 0<t \leq 10 \\ \frac{10}{2+\cos t}, & t>10\end{cases}
$$

Obviously, $f$ is a piecewise function. The analytical solution is not available for this example.

We use the developed KDC-GFDM(2) and the COMSOL software for solving this problem. The KDC-GFDM(2) uses 11081 collocation points which can be seen in Fig. 12(b). The time step size is set to $\Delta t=0.5$, and 3 Gaussian nodes in $\Delta t$. The COMSOL employs 14809 tetrahedron elements and the time step of 0.1. Fig. 13 displays the curves of numerical results of points $A(0.5,0.25,0)$ and $B(-0.5,-0.15,-0.1)$ from $t=0$ to $t=20$ calculated by the KDC-GFDM(2) and the COMSOL software. The agreements of numerical results demonstrate that the developed scheme can accurately describes the wave propagation. 


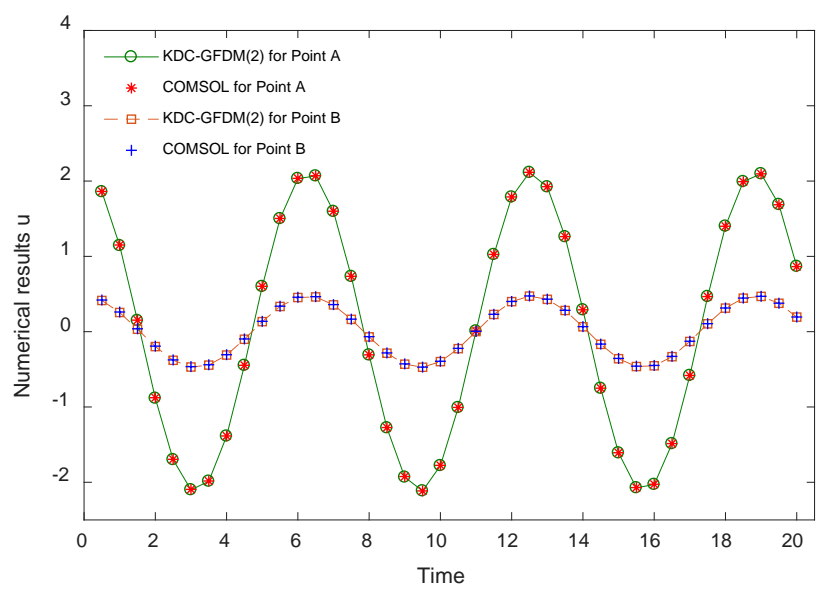

Figure 13: Numerical results $u$ obtained by the KDC-GFDM(2) and the COMSOL software.

\section{Conclusions and generalization}

This paper constructs a numerical framework for simulating wave propagation phenomena in long-time intervals by taking full advantages of two approaches, i.e., the KDC technique and the GFDM. The wave equation is transformed to the modified Helmholtz equation at Gaussion nodes in temporal direction through the KDC method. Then the modified Helmholtz equation in space is solved by using the GFDM with the second and fourth order approximations. 2D and 3D wave problems with different conditions and geometries are investigated by the present numerical framework, and numerical results demonstrate that: a) Large size of time step can be allowed to implement the KDCGFDM; b) Numerical errors is stable when using the KDC-GFDM for simulating wave propagation phenomena in long-time intervals; c) The KDC-GFDM has a good performance for the solution of the problem with the complicated geometry and the piecewise function of the source term.

In view of the above-mentioned advantages of the KDC-GFDM, the further application using the developed approach for coupled acoustic-structural analysis will be addressed in subsequent report.

\section{Acknowledgements}

The work described in this paper was supported by the National Natural Science Foundation of China (Grant No. 11802165), the Natural Science Foundation of Shandong Province of China (Grant No. ZR2017BA003), and the China Postdoctoral Science Foundation (Grant No. 2019M650158). 


\section{References}

[1] N.-J. WU, T.-K. TSAY, AND D. L. YOUNG, Meshless numerical simulation for fully nonlinear water waves, Int. J. Numer. Methods Fluids, 50 (2006), pp. 219-234.

[2] W. QU, W. CHEN, AND C. ZHENG, Diagonal form fast multipole singular boundary method applied to the solution of high-frequency acoustic radiation and scattering, Int. J. Numer. Methods Eng., 111 (2017), pp. 803-815.

[3] X. YOU, W. LI, AND Y. CHAI, A truly meshfree method for solving acoustic problems using local weak form and radial basis functions, Appl. Math. Comput., 365 (2020), pp. 124694.

[4] J. LI, L. ZHANG, AND Q.-H. QIN, A regularized method of moments for three-dimensional timeharmonic electromagnetic scattering, Appl. Math. Lett., 112 (2021), pp. 106746.

[5] L. QIU, C. HU, AND Q.-H. QIN, A novel homogenization function method for inverse source problem of nonlinear time-fractional wave equation, Appl. Math. Lett., (2020), pp. 106554.

[6] G. FAIRWEATHER, AND A. KARAGEORGHIS, The method of fundamental solutions for elliptic boundary value problems, Adv. Comput. Math., 9 (1998), pp. 69.

[7] F. WANG, C.-M. FAN, Q. HUA, AND Y. GU, Localized MFS for the inverse Cauchy problems of two-dimensional Laplace and biharmonic equations, Appl. Math. Comput., 364 (2020), pp. 124658.

[8] F. WANG, C.-M. FAN, C. ZHANG, AND J. LIN, A localized space-time method of fundamental solutions for diffusion and convection-diffusion problems, Adv. Appl. Math. Mech., 12 (2020), pp. 940-958.

[9] C.-S. CHEN, C.-M. FAN, AND P. WEN, The method of approximate particular solutions for solving elliptic problems with variable coefficients, Int. J. Comput. Methods, 8 (2011), pp. 545559.

[10] Q. XI, C. S. CHEN, Z. FU, AND E. COMINO, The MAPS with polynomial basis functions for solving axisymmetric time-fractional equations, Comput. Math. Appl., (2019), https://doi.org/10.1016/j.camwa.2019.1011.1014.

[11] L. CHEN, X. LIU, AND X. LI, The boundary element-free method for 2D interior and exterior Helmholtz problems, Comput. Math. Appl., 77 (2019), pp. 846-864.

[12] L. CHEN, AND X. LI, A complex variable boundary element-free method for the Helmholtz equation using regularized combined field integral equations, Appl. Math. Lett., 101 (2020), pp. 106067.

[13] J. Lin, C. ZHANG, L. SUN, AND J. LU, Simulation of seismic wave scattering by embedded cavities in an elastic half-plane using the novel singular boundary method, Adv. Appl. Math. Mech., 10 (2018), pp. 322-342.

[14] A. ZHANG, Y. GU, Q. HuA, W. CHEN, AND C. ZHANG, A regularized singular boundary method for inverse Cauchy problem in three-dimensional elastostatics, Adv. Appl. Math. Mech., 10 (2018), pp. 1459-1477.

[15] W. QU, AND W. CHEN, Solution of two-dimensional stokes flow problems using improved singular boundary method, Adv. Appl. Math. Mech., 7 (2015), pp. 13-30.

[16] J. LI, W. CHEN, Q.-H. QIN, AND Z. FU, A modified multilevel algorithm for large-scale scientific and engineering computing, Comput. Math. Appl., 77 (2019), pp. 2061-2076.

[17] W. LI, A fast singular boundary method for 3D Helmholtz equation, Comput. Math. Appl., 77 (2019), pp. 525-535.

[18] X. WeI, A. HuANG, AND L. Sun, Singular boundary method for 2D and 3D heat source reconstruction, Appl. Math. Lett., 102 (2020), pp. 106103.

[19] Z. FU, Q. XI, Y. LI, H. HUANG, AND T. RABCZUK, Hybrid FEM-SBM solver for structural vibration induced underwater acoustic radiation in shallow marine environment, Comput. Methods 
Appl. Mech. Eng., 369 (2020), pp. 113236.

[20] M. DEHGHAN, AND A. SHOKRI, A meshless method for numerical solution of the one-dimensional wave equation with an integral condition using radial basis functions, Numer. Algorithms, 52 (2009), pp. 461.

[21] Z. ZHANG, D.-M. Li, Y.-M. CHENG, AND K. M. LIEW, The improved element-free Galerkin method for three-dimensional wave equation, Acta Mech. Sinica, 28 (2012), pp. 808-818.

[22] Z. WANG, J. LI, B. WANG, Y. XU, AND X. CHEN, A new central compact finite difference scheme with high spectral resolution for acoustic wave equation, J. Comput. Phys., 366 (2018), pp. 191-206.

[23] F. UREN̈A, J. J. Benito, E. SAlete, AND L. GAVEte, A note on the application of the generalized finite difference method to seismic wave propagation in 2D, J. Comput. Appl. Math., 236 (2012), pp. 3016-3025.

[24] W. QU, Y. GU, Y. ZHANG, C. M. FAN, AND C. ZHANG, A combined scheme of generalized finite difference method and Krylov deferred correction technique for highly accurate solution of transient heat conduction problems, Int. J. Numer. Methods Eng., 117 (2019), pp. 63-83.

[25] F. U. PRieto, J. J. BEnito MuÑoz, AND L. G. CORVINOS, Application of the generalized finite difference method to solve the advection-diffusion equation, J. Comput. Appl. Math., 235 (2011), pp. 1849-1855.

[26] C.-M. FAN, C.-N. CHU, B. ŠARLER, AND T.-H. LI, Numerical solutions of waves-current interactions by generalized finite difference method, Eng. Anal. Boundary Elements, 100 (2019), pp. 150-163.

[27] W. QU, A high accuracy method for long-time evolution of acoustic wave equation, Appl. Math. Lett., 98 (2019), pp. 135-141.

[28] Y. WANG, Y. GU, AND J. LIU, A domain-decomposition generalized finite difference method for stress analysis in three-dimensional composite materials, Appl. Math. Lett., 104 (2020), pp. 106226.

[29] Z.-J. FU, Z.-Y. XIE, S.-Y. JI, C.-C. TSAI, AND A.-L. LI, Meshless generalized finite difference method for water wave interactions with multiple-bottom-seated-cylinder-array structures, Ocean Eng., 195 (2020), pp. 106736.

[30] P.-W. LI, Z.-J. FU, Y. GU, AND L. SONG, The generalized finite difference method for the inverse Cauchy problem in two-dimensional isotropic linear elasticity, Int. J. Solids Structures, 174-175 (2019), pp. 69-84.

[31] W. QU, AND H. HE, A spatial-temporal GFDM with an additional condition for transient heat conduction analysis of FGMs, Appl. Math. Lett., 110 (2020), pp. 106579.

[32] J. Jia, J. C. HiLl, K. J. Evans, G. I. FANN, AND M. A. TAYLOR, A spectral deferred correction method applied to the shallow water equations on a sphere, Monthly Weather Rev., 141 (2013), pp. 3435-3449.

[33] A. Dutt, L. Greengard, And V. RokHlin, Spectral Deferred Correction Methods for Ordinary Differential Equations, BIT Numer. Math., 40 (2000), pp. 241-266.

[34] A. T. LAYTON, AND M. L. MINION, Conservative multi-implicit spectral deferred correction methods for reacting gas dynamics, J. Comput. Phys., 194 (2004), pp. 697-715.

[35] A. Christlieb, B. ONG, AND J.-M. QIU, Integral deferred correction methods constructed with high order Runge-Kutta integrators, Math. Comput., 79 (2010), pp. 761-783.

[36] J. HuANG, J. JiA, AND M. MinION, Accelerating the convergence of spectral deferred correction methods, J. Comput. Phys., 214 (2006), pp. 633-656.

[37] J. HuAng, J. JiA, AND M. Minion, Arbitrary order Krylov deferred correction methods for differential algebraic equations, J. Comput. Phys., 221 (2007), pp. 739-760. 
[38] J. JiA, AND J. HUANG, Krylov deferred correction accelerated method of lines transpose for parabolic problems, J. Comput. Phys., 227 (2008), pp. 1739-1753.

[39] W. Qu, N. BRANDON, D. CHEN, J. HuANG, AND T. KRESS, A numerical framework for integrating deferred correction methods to solve high order collocation formulations of ODEs, J. Sci. Comput., 68 (2016), pp. 484-520.

[40] D. A. KNOLL, AND D. E. KEYES, Jacobian-free Newton-Krylov methods: a survey of approaches and applications, J. Comput. Phys., 193 (2004), pp. 357-397.

[41] W. QU, C.-M. FAN, AND Y. ZHANG, Analysis of three-dimensional heat conduction in functionally graded materials by using a hybrid numerical method, Int. J. Heat Mass Transfer, 145 (2019), pp. 118771.

[42] T. BelytschKo, Y. Krongauz, D. Organ, M. Fleming, AND P. KrysL, Meshless methods: an overview and recent developments, Comput. Methods Appl. Mech. Eng., 139 (1996), pp. 347.

[43] J. SLADeK, V. SLADEK, AND Y. C. HON, Inverse heat conduction problems by meshless local Petrov-Galerkin method, Eng. Anal. Bound. Elem., 30 (2006), pp. 650-661.

[44] W. QU, C.-M. FAN, AND X. LI, Analysis of an augmented moving least squares approximation and the associated localized method of fundamental solutions, Comput. Math. Appl., 80 (2020), pp. 13-30.

[45] T. ZHU, J.-D. ZHANG, AND S. N. ATLURI, A local boundary integral equation (LBIE) method in computational mechanics, and a meshless discretization approach, Comput. Mech., 21 (1998), pp. 223-235.

[46] C. M. FAn, Y. K. Huang, P. W. Li, AND C. L. CHIU, Application of the generlized finite-difference method to inverse biharmonic boundary-value problems, Numer. Heat Tranf. BFundam., 65 (2014), pp. 129-154.

[47] X. LI, AND H. DONG, Error analysis of the meshless finite point method, Appl. Math. Comput., $382(2020) 125326$.

[48] T. ZHANG, AND X. LI, Analysis of the element-free Galerkin method with penalty for general second-order elliptic problems, Appl. Math. Comput., 380 (2020), pp. 125306.

[49] X. LI, AND H. DONG, An element-free Galerkin method for the obstacle problem, Appl. Math. Lett., 112 (2021), pp. 106724.

[50] Q. XI, Z. FU, W. WU, H. WANG, AND Y. WANG, A novel localized collocation solver based on Trefftz basis for potential-based inverse electromyography, Appl. Math. Comput., 390 (2021), pp. 125604.

[51] W. QU, W. CHEN, AND Y. GU, Fast multipole accelerated singular boundary method for the 3D Helmholtz equation in low frequency regime, Comput. Math. Appl., 70 (2015), pp. 679-690.

[52] E. HAIRER, C. LuBICH, AND G. WANNER, Geometric Numerical Integration: StructurePreserving Algorithms for Ordinary Differential Equations, Springer Science \& Business Media, 2006. 